\title{
Management der
}

\section{Hyperglykämie bei Typ-2-Diabetes}

Bei Typ-2-Diabetikern könne durch normnahe Blutglukosewerte mit einem $\mathrm{HbA}_{1 c}$-Zielwert unter 7\% ein großer Teil mikrovaskulärer und neuropathischer Erkrankungen verhindert werden, so die Autoren. Die meisten Patienten sollten nach folgendem Algorithmus behandelt werden.

\section{Schritt 1: Lebensstiländerung und Metformin}

Bei Manifestation eines Typ-2-Diabetes sollte an erster Stelle eine Lebensstiländerung mit Optimierung des Ernährungs- und Bewegungsverhaltens initiiert werden. Dies gelte auch für die 10-20\% normalgewichtigen Patienten. Darüber hinaus sollte ebenfalls bereits bei Diagnosestellung mit der Titration von Metformin begonnen werden, sofern keine Kontraindikationen beständen (s. Infokasten). Die rasche Kombination mit einem zweiten oralen Antidiabetikum könne erwogen werden, wenn Symptome einer Hyperglykämie anhielten.

\section{Schritt 2: Kombinationstherapie}

Falls mit Lebensstiländerung und maximal tolerierter Metformindosis das Therapieziel nicht oder nicht auf Dauer

\section{Titration von Metformin}

1. Beginn mit Metformin in niedriger Dosierung entweder $500 \mathrm{mg} 1-2 \times / \mathrm{d}$ zum Frühstück und/oder Abendessen oder $850 \mathrm{mg} 1 \times / \mathrm{d}$.

2. Nach 5-7 Tagen, sofern keine gastrointestinalen Nebenwirkungen aufgetreten sind, Dosiserhöhung auf $850 \mathrm{mg}$ oder 2 Tabletten á $500 \mathrm{mg} 2 \times / \mathrm{d}$ vor dem Frühstück und/oder Abendessen.

3. Falls im Rahmen der Dosissteigerung gastrointestinale Nebenwirkungen auftreten, Reduktion auf die vorherige Dosis und erneuter Versuch einer Dosissteigerung zu einem späteren Zeitpunkt.

4. Die effektive Maximaldosierung kann $1000 \mathrm{mg} 2 \times / \mathrm{d}$ betragen, oft liegt sie bei $850 \mathrm{mg} 2 \times / \mathrm{d}$. Eine gering stärkere Wirksamkeit wurde bei einer Dosis von $2500 \mathrm{mg}$ pro Tag beobachtet. Gastrointestinale Nebenwirkungen können die maximal verträgliche Dosis limitieren.

5. Unter Kostenaspekten ist generisches Metformin die Therapie der ersten Wahl. erreicht werden könne, sollte zusätzlich zu Metformin ein weiteres Medikament gegeben werden. Es bestehe Konsensus, vorrangig mit Sulfonylharnstoff ode vor allem bei $\mathrm{HbA}_{1 \mathrm{c}}$-Werten über - mit basalem Insulin zu kombinie

\section{Schritt 3: Weiteres Vorgehen}

Falls mit Lebensstiländerung, Metformin und entweder Sulfonylharnstoff oder basalem Insulin das Therapieziel nicht oder nicht auf Dauer erreicht werden könne, sollte im nächsten Schritt eine Insulintherapie begonnen oder die bisherige Therapie intensiviert werden. Mit Beginn der Insulininjektionen müssten insulinotrope Substanzen (Sulfonylharnstoffe, Glinide) reduziert und schließlich abgesetzt werden. Die Kombination von 3 oralen Antidiabetika könne nicht empfohlen werden, da sie nicht effektiver aber kostenintensiver sei als der Beginn oder die Intensivierung einer Insulintherapie.

\section{Weniger gut validierter Algorithmus}

Nach Ansicht der Autoren sollte die Kombination von Metformin mit entweder Exenatid oder Pioglitazon nur in ausgewählten Fällen erwogen werden, z.B. wenn Hypoglykämien aufgrund der Arbeitsplatzsituation zu Gefährdungen führen könnten. Exenatid käme insbesondere dann in Frage, wenn im Vordergrund eine Gewichtsreduktion stehe und der $\mathrm{HbA}_{1 \mathrm{c}}$-Wert unter $8 \%$ liege.

Dr. med. Winfried Keuthage, Münster

Quelle: Nathan DM, Buse JB, Davidson MB et al. Medical Management of Hyperglycemia in Type 2 Diabetes: A Consensus Algorithm for the Initiation and Adjustment of Therapy. Diabetes Care 2009; 32: 193-203

1 European Association for the Study of Diabetes 2 Action in Diabetes and Vascular Disease Preterax and Diamicron Modified Release Controlled Evaluation

${ }^{3}$ Action to Control Cardiovascular Risk in Diabetes

${ }^{4}$ Veteran Affairs Diabetes Trial

${ }^{5}$ American Diabetes Association

${ }^{6}$ American College of Cardiology Foundation

7 American Heart Association

${ }^{8}$ Diabetes Control and Complications Trial

${ }^{9}$ UK Prospective Diabetes Study 